\title{
Homeopathy and Quantum Field Theory
}

\author{
Otto Weingärtner \\ Dr. Reckeweg \& Co., Bensheim, Germany
}

Apart from Axiomatic Quantum Field Theory which is no longer as important as it was in the 1970s, Quantum Field Theory (QFT) is a general term for theories that describe interactions between particles. In many cases fundamentals of a QFT can be traced back to a combination of methods of classical Field Theory and/or Quantum Mechanics. Mostly Relativity is considered, too. The most outstanding feature of QFT is the so-called second quantization. While quantization in normal Quantum Mechanics is a computational procedure which describes the process of quantization of energy and momentum of particles, second quantization in QFT additionally describes the interaction between particles in terms of virtual quanta. This is done for instance with the help of creation- and annihilation-operators, Feynman graphs, path integrals, renormalization etc. QFTs which describe reality are gauge theories. The perfect example of a QFT is Quantum Electrodynamics which describes among others the interaction between charged particles by the interchange of virtual photons. The force being responsible for phenomena within Quantum Electrodynamics is the electromagnetic force. Together with characterizations belonging to the weak force and the strong force QFTs can be formulated which describe all known particles and phenomena under the roof of a so-called standard model. The ultimate goal of today's physics is to unify elementary particle physics and gravitation which is ruled by another force, the gravitational one. The QFT belonging to this is sometimes called the grand unified theory and it is not completed yet.

As to the language in which QFTs should be formulated, Galilei's words 'Il libro de la natura é scritto in lingua mathematica' are as relevant as they always have been.

Following this, it is important to notice that Patient-PractitionerRemedy (PPR) entanglement is on the one hand just a metaphor with no experimental verification. On the other hand PPR entanglement adopts the quantum mechanical formalism by suggesting identical behavior of wave functions $\Psi$ in the Eigenvalue-equation $\mathrm{H} \Psi=\mathrm{E} \Psi$ and PPR functions $\Psi_{\mathrm{PPR}}=\mathrm{a} \Psi_{\mathrm{Px}}+\mathrm{b} \Psi_{\mathrm{Pr}}+\mathrm{c} \Psi_{\mathrm{Rx}}$. Keeping in mind this metaphorical use of the formalism of quantum mechanics the readership of Milgrom's former articles on PPR entanglement has got an excellent opportunity to fix homeopathic ideas for themselves within a kind of a meta-mathematical lan- guage. For a lot of people this is certainly helpful in sorting things out and making presumptions a bit more precise.

Milgrom's latest paper on a model of homeopathic action based on QFT [1, in this issue] is an additional step in the metaphorical use of facts from Quantum Physics.

After a very clear and elucidative first half of the paper, summarizing features of QFT, Milgrom continues in the second part of the paper with 'Quantum field theory as a metaphor for the homeopathic process: quantum homeodynamics (QHD)'. Here physicists would expect precise definitions of states, of operators representing observables, of domains and ranges of operators, of tensor-products and all that, but would be aware of the considerable amount of technical difficulties. Milgrom simply inserts PPRwords in his previous verbal explanations of some ideas of QFT. So, his article remains a somewhat speculative but nevertheless astonishing list of similarities between quantum field theoretical considerations and homeopathy which is certainly worthwhile to be picked up again by physicists. The latter, however, would mean that particles (or something with particle-like characteristics which can play the role of particles) become specified. This would mean, that interactions between these particles become specified. Last but no least this would mean that the fields originating from these particles are not in contradiction with the idea of a field in normal QFT. Even these difficulties which arise when the entanglement metaphor is intended to be applied to quantum field phenomena in order to develop a model of homeopathic action are worthwhile to be discussed. As a first step towards a successful attack on these problems the role of entanglement in normal QFT should be investigated in the light of the ideas of Weak Quantum Theory as they have been presented by Atmanspacher et al. [2].

\section{References}

1 Milgrom LR: Towards a new model of the homeopathic process based on Quantum Field Theory. Forsch Komeplementärmed DOI: 10.1159/000093662.

2 Atmanspacher H, Römer H, Walach H: Weak quantum theory, complementarity and entanglement in physics and beyond. Found Phys 2002;32:379-406.

\begin{tabular}{ll}
\hline KARGER & ๑ 2006 S. Karger GmbH, Freiburg \\
Fax +49 76145207 14 & Accessible online at: \\
$\begin{array}{l}\text { E-mail Information@Karger.de } \\
\text { www.karger.com }\end{array}$ & www.karger.com/fok
\end{tabular}

